Faculty of Sciences and Mathematics, University of Niš, Serbia

Available at: http://www.pmf.ni.ac.yu/filomat

Filomat 23:2 (2009), 43-55

\title{
AN APPLICATION OF GROEBNER BASES TO PLANARITY OF INTERSECTION OF SURFACES
}

\author{
Branko Malešević*, Marija Obradović
}

\begin{abstract}
In this paper we use Groebner bases theory in order to determine planarity of intersections of two algebraic surfaces in $\mathbf{R}^{3}$. We specially considered plane sections of certain type of conoid which has a cubic egg curve as one of the directrices. The paper investigates a possibility of conic plane sections of this type of conoid.
\end{abstract}

\section{THE BASIC CONCEPT OF GROEBNER BASES}

Many problems in mathematics can be treated as a problem of solvability of a system of polynomial equations:

$$
\begin{gathered}
f_{1}\left(x_{1}, x_{2}, \ldots, x_{n}\right)=0 \\
f_{2}\left(x_{1}, x_{2}, \ldots, x_{n}\right)=0 \\
\vdots \\
f_{m}\left(x_{1}, x_{2}, \ldots, x_{n}\right)=0
\end{gathered}
$$

where $f_{1}, f_{2}, \ldots, f_{m}$ are polynomials in $n$ variables over set of complex numbers $\mathbf{C}$. The main matter in the theory of systems of polynomial equations are Groebner bases. Let us define this concept through following considerations. The polynomial of several variables:

$$
P\left(x_{1}, \ldots, x_{n}\right)=\sum_{\alpha \in A_{0}} c_{\alpha} x_{1}^{\alpha_{1}} \cdots x_{n}^{\alpha_{n}}
$$

is considered as a sum over $n$-tuples $\alpha=\left(\alpha_{1}, \ldots, \alpha_{n}\right)$ from a finite set $A_{0} \subset \mathbf{N}_{0}^{n}\left(c_{\alpha} \neq 0\right)$. The term $x_{1}^{\alpha_{1}} \cdots x_{n}^{\alpha_{n}}$ is called the monomial, while the summand $c_{\alpha} x_{1}^{\alpha_{1}} \cdots x_{n}^{\alpha_{n}}$ is called the term of the polynomial.

*First author supported in part by the project MNTRS, Grant No. ON144020.

2000 Mathematics Subject Classifications. 14N05, 51N35.

Key words and Phrases. Groebner bases, egg curve based conoid, planar intersection. 
A monomial order $\succ$ is a total order on the set of all monomials, satisfying two additional properties: the ordering respects multiplication (if $u \succ v$ and $w$ is any other monomial, then $u w \succ v w$ ) and the ordering is a well ordering. The lexicographic order over a set of monomials $\succ_{\text {lex }}$ is defined by order of variables $x_{1} \succ_{\text {lex }} \ldots \succ_{\text {lex }} x_{n}$ so that: $x_{1}^{\alpha_{1}} \cdots x_{n}^{\alpha_{n}} \succ_{l e x} x_{1}^{\beta_{1}} \cdots x_{n}^{\beta_{n}}$ iff $\alpha_{1}=\beta_{1}, \ldots, \alpha_{k-1}=\beta_{k-1}, \alpha_{k}>\beta_{k}$ for some $k$. Other types of monomial ordering are presented in [1]. Let us notice that polynomial (2) can be arranged in the given monomial order, so that the first term in the sum $f$ is the leading term and denoted by $L T(f)$. Then leading term is shown as the product of the leading coefficient $L C(f)$ and the leading monomial $L M(f)$, i.e. $L T(f)=L C(f) \cdot L M(f)$.

In the polynomial ring $\mathbf{C}\left[x_{1}, \ldots, x_{n}\right]$ we determine a division algorithm, which follows. Let a $m$-tuple of polynomials $F=\left(f_{1}, \ldots, f_{m}\right)$ be given, arranged into monomial order $\succ$ over the set of monomials. Then we quote an division algorithm with the following pseudo-code [2]:

$$
\begin{array}{ll}
\text { Input: } & f, f_{1}, \ldots, f_{m} \\
\text { Output: } & r, a_{1}, \ldots, a_{m} \\
& r:=0, a_{1}:=0, \ldots, a_{m}:=0 \\
& p:=f \\
\text { WHILE } p \neq 0 \text { DO } & \\
i:=1 & \\
\text { divisionoccured }:=\text { false } \\
\text { WHILE } i \leq m \text { AND divisionoccured }=\text { false DO } \\
\text { IF } L T\left(f_{i}\right) \text { divides } L T(p) \text { THEN } \\
a_{i}:=a_{i}+L T(p) / L T\left(f_{i}\right) \\
p:=p-L T(p) / L T\left(f_{i}\right) \cdot f_{i} \\
\text { divisionoccured }:=\text { true } \\
\text { ELSE } \\
i:=i+1 \\
\text { IF divisionoccured }=\text { false THEN } \\
r:=r+L T(p) \\
\quad p:=p-L T(p)
\end{array}
$$

Using the previous algorithm, every polynomial $f \in \mathbf{C}\left[x_{1}, \ldots, x_{n}\right]$ can be presented as follows:

$$
f=a_{1} f_{1}+\ldots+a_{m} f_{m}+r
$$

for some $a_{i}, r \in \mathbf{C}\left[x_{1}, \ldots, x_{n}\right](i=1, \ldots, m)$, while either $r=0$ or $r$ is $\mathbf{C}$-linear combination of monomials none of which is divisible by any $L T\left(f_{1}\right), \ldots, L T\left(f_{m}\right)$. The polynomial $r=R E M(f, F)$ is called the remainder of division of $f$ by $m$-tuple of polynomials $F$ arranged into monomial order $\succ$. The main characteristic of the remainder $r$ is that it is not uniquely determined in comparison to order of division by polynomials from $m$-tuple $F$. Let us remark:

$$
L T(f) \succ L T(r) .
$$


For a $m$-tuple of polynomials $F=\left(f_{1}, \ldots, f_{m}\right)$ the ideal is determined by:

$$
I=\left\{\sum_{i=1}^{m} h_{i} f_{i} \mid h_{1}, \ldots, h_{m} \in \mathrm{C}\left[x_{1}, \ldots, x_{n}\right]\right\} .
$$

It is denoted by $\left\langle f_{1}, \ldots, f_{m}\right\rangle$. Then polynomials $f_{1}, \ldots, f_{m}$ are called a generators for the ideal $I=\left\langle f_{1}, \ldots, f_{m}\right\rangle$ in the polynomial ring $\mathbf{C}\left[x_{1}, \ldots, x_{n}\right]$. Let us emphasize that Hilbert Basis Theorem claims that every ideal has a finite generating set [2]. Next, for the ideal $I=\left\langle f_{1}, \ldots, f_{m}\right\rangle$ we define the ideal of leading terms:

$$
\langle L T(I)\rangle=\langle\{L T(f) \mid f \in I\}\rangle .
$$

In any ideal $I=\left\langle f_{1}, \ldots, f_{m}\right\rangle$ it is true:

$$
\left\langle L T\left(f_{1}\right), \ldots, L T\left(f_{m}\right)\right\rangle \subseteq\langle L T(I)\rangle
$$

Generating set $G=\left\{g_{1}, \ldots, g_{s}\right\}$ of the ideal $I=\left\langle f_{1}, \ldots, f_{m}\right\rangle$ is called a Groebner basis if

$$
\left\langle L T\left(g_{1}\right), \ldots, L T\left(g_{s}\right)\right\rangle=\langle L T(I)\rangle
$$

Let us remark that for each permutation of $s$-tuple $G=\left\langle g_{1}, \ldots, g_{s}\right\rangle$ the remainder $r=R E M(f, G)$ is uniquely determined [2].

B. BuChBERGER in his dissertation has shown that for each ideal there exists a Groebner basis by following algorithm [2]:

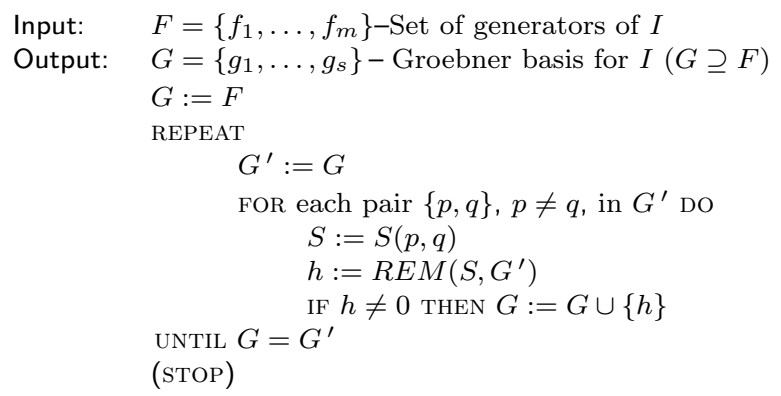

In the previous algorithm we used the $S$-polynomial of $f$ and $g$ (in relation to the fixed monomial order $\succ$ ) which is defined by:

$$
S=S(f, g)=\frac{L C M(L M(f), L M(g))}{L T(f)} \cdot f-\frac{L C M(L M(f), L M(g))}{L T(g)} \cdot g,
$$

where $L C M$ is the least common multiple. For different sets of generators of the ideal $I$ Groebner basis, produced by the Buchberger's algorithm, is not unique. Groebner basis is minimal iff $L C(g)=1$ for all $g \in G$, whereat $\{L T(g) \mid g \in G\}$ is a minimal basis of the monomial ideal $\langle\{L T(f) \mid f \in I\}\rangle$ (see Exercise 6/§7/Ch. $2[2]$ ). Minimal Groebner basis is not unique as well. Reduced Groebner basis is minimal Groebner basis for the ideal $I$ such that for all $p \in G$ there is no monomial of $p$ which lies in $\langle\{L T(g) \mid g \in G \backslash\{p\}\}\rangle$ [1], [2]. The following statement is true [2]: 
Theorem 1.1. Every polynomial ideal has a unique reduced Groebner basis, related to a fixed monomial order.

Groebner bases in applications are usually considered in reduced form, as it is emphasized in [2] and [15]. In the next part of this paper we consider an application of the Groebner bases in the real three-dimensional geometry.

\section{AN APPLICATION OF GROEBNER BASES TO PLANARITY OF INTERSECTION OF SURFACES}

Let us consider the consistent system of two real non-linear polynomial equations with three variables:

$$
f_{1}(x, y, z)=0 \wedge f_{2}(x, y, z)=0 .
$$

For the system (12) we define that the system has planar solution if there exists a linear-polynomial:

$$
g=g(x, y, z)=A x+B y+C z+D,
$$

for some real constants $A, B, C, D$, such that every solution of the system (12) is also a solution of the linear equation:

$$
g(x, y, z)=0 .
$$

Thus we indicate that the system has planar intersection. Let a monomial order be determined, then the following statement is true:

Theorem 2.1. If a Groebner basis of the system (12) contains the linear polynomial (13), then the solution of the system (12) is planar.

The following statement is formulated for lexicographic order $x \succ_{\text {lex }} y \succ_{\text {lex }} z$ :

Theorem 2.2. Let the system (12) have the planar intersection by:

$$
A x+B y+C z+D=0,
$$

for some $A, B, C, D \in \mathbf{R}$ and $A \neq 0$. If for the ideal $I=\left\langle f_{1}, f_{2}\right\rangle$ the following is true:

$$
y \notin\langle L T(I)\rangle \wedge z \notin\langle L T(I)\rangle,
$$

then the linear polynomial:

$$
\hat{g}=\hat{g}(x, y, z)=x+(B / A) y+(C / A) z+(D / A)
$$

is an element of the reduced Groebner basis related to lexicographic order $\succ_{l e x}$. 
Proof. The system (12) is equivalent with the following system:

$$
f_{1}(x, y, z)=0 \wedge f_{2}(x, y, z)=0 \wedge \hat{g}(x, y, z)=0 .
$$

The reduced Groebner bases for systems (12) and $\left(12^{\prime}\right)$ are equal. Let us prove that $\hat{g} \in I$ is element of reduced Groebner basis for system $\left(12^{\prime}\right)$. Using Buchberger algorithm, we can assume that polynomial $\hat{g}$ is element of the Groebner basis $G_{1}$. Let us consider minimal Groebner basis $G_{\min }$ formed from $G_{1}$ such that $\hat{g} \in G_{\min }$. Next, let us prove that polynomial $\hat{g}$ is reduced related to $G_{\min }$ (see Proposition 6./\$7/Ch. 2 in [2] or Lect. 14 in [16]). By condition (16) it follows that for a linear polynomial $\hat{g}=x+(B / A) y+(C / A) z+(D / A)$ it is true that $x, y, z \notin\left\langle L T\left(G_{\min } \backslash\{\hat{g}\}\right)\right\rangle$. If polynomial $\hat{g}$ is reduced in one minimal Groebner basis, then $\hat{g}$ is reduced related to every minimal Groebner basis (see proof of the Proposition $6 . / \S 7 /$ Ch. 2 in [2]) and therefore polynomial $\hat{g}$ is an element of the unique reduced Groebner basis.

Let us emphasize that computer algebra systems compute reduced Groebner basis. In this paper we use computer algebra system Maple and Maple Package "Groebner" which is initiated by:

$$
\text { with(Groebner); }
$$

Let $F:=\left[f_{1}, f_{2}\right]$ be the list of two polynomials of three variables $x, y, z$. In Maple lexicographic order $\mathrm{x} \succ_{\text {lex }} \mathrm{y} \succ_{\text {lex }} \mathrm{z}$ we denote by $\operatorname{plex}(\mathrm{x}, \mathrm{y}, \mathrm{z})$. Then by command

$$
\operatorname{Basis}(\mathrm{F}, \operatorname{plex}(\mathrm{x}, \mathrm{y}, \mathrm{z}))
$$

Maple computes the reduced Groebner basis. If some variables are omitted from the list of variables, then the algorithm considers these variables as non-zero constants (see Appendix $\mathrm{C} / \S 2$ (Maple) in [2] and [13], [14]).

Example 2.3. Let the system of two polynomials be given:

$$
\mathrm{F}:=\left[\mathrm{z}-\frac{\mathrm{x}^{2}}{\mathrm{a}^{2}}-\frac{\mathrm{y}^{2}}{\mathrm{~b}^{2}}, \frac{\mathrm{x}^{2}}{\mathrm{a}^{2}}+\frac{\mathrm{y}^{2}}{\mathrm{~b}^{2}}-\frac{\mathrm{x}}{\mathrm{a}}-\frac{\mathrm{y}}{\mathrm{b}}\right]
$$

where $\mathrm{x}, \mathrm{y}, \mathrm{z}$ are variables and $\mathrm{a}, \mathrm{b}$ are non-zero constants. We compute reduced Groebner basis using Maple by command Basis $(\mathrm{F}, \operatorname{plex}(\mathrm{x}, \mathrm{y}, \mathrm{z}))$; and the result is the following list of the (arranged) polynomials:

$$
\left[b x+a y-a b z, 2 y^{2}-2 b y z-b^{2} z^{2}-b^{2} z\right]
$$

Let us emphasize that the answer is the reduced Groebner basis, except for clearing denominators (so leading coefficients of the Groebner basis are polynomials in a and $\mathrm{b})[2]$. This proves that curve of intersection lies in the following plane:

$$
\mathrm{bx}+\mathrm{ay}-\mathrm{abz}=0 .
$$

Let us remark that $\langle L T(I)\rangle=\left\langle\mathrm{bx}, 2 \mathrm{y}^{2}\right\rangle$ and the condition (16) is fulfilled. 
Example 2.4. Let the system of two polynomials be given:

$$
F:=\left[x+y z+y-z^{4}-4, y-z^{3}-1\right]
$$

where $\mathrm{x}, \mathrm{y}, \mathrm{z}$ are variables. We compute reduced Groebner basis using Maple by command $\operatorname{Basis}(\mathrm{F}, \operatorname{plex}(\mathrm{x}, \mathrm{y}, \mathrm{z}))$; and the result is the following list of the (arranged) polynomials:

$$
\left[x+z^{3}+z-3, y-z^{3}-1\right]
$$

Note that:

$$
1 \cdot\left(x+y z+y-z^{4}-4\right)-z \cdot\left(y-z^{3}-1\right)=x+y+z-4
$$

and therefore the intersection is planar. Let us emphasize that $\langle L T(I)\rangle=\langle\mathrm{x}, \mathrm{y}\rangle$ and condition (16) is not fulfilled.

Remark 2.5. It is possible to formulate and prove the previous theorems in more extended sense than in the given three-dimensional formulations and lexicographic order.

\section{AN APPLICATION OF THE GROEBNER BASES TO PLANE SECTIONS OF ONE TYPE OF CONOID WITH BASIC CUBIC EGG CURVE}

In this part, there will be considered an application of the Groebner bases theory on a surface which is obtained by motion of the system of generatrices along three directrices.

Let us accept cubic egg curve $g$, the right cubic hyperbolic parabola of type $A$ [3] which lies in the plane $x-y$, for the initial curve taken as a plane section of a conoid, and also for its directrix. Starting from the definition of conoid [6] (p. 277) as a surface which has two directrices in finiteness: plane curve $d_{1}$, and straight line $d_{2}$, while the third directrix, straight line $d_{3}$, lies in infinity, we consider a conoid with [11]:

(i) Cubic curve $(g)$ in the form $g=d_{1}: b^{2} x^{2}+a^{2} y^{2}+2 d x y^{2}+d^{2} y^{2}-a^{2} b^{2}=0$ as directrix $d_{1}(a>b>0, a-b \geq d>0)$.

(ii) Straight line parallel to the axis $y$, in the plane $y-z$ on elevation $z=h$ as directrix $d_{2}(h>0)$.

(iii) Plane $x-z$ as directrix plane of the conoid, and its infinitely distant straight line as directrix $d_{3}$, in order to obtain a right conoid. 


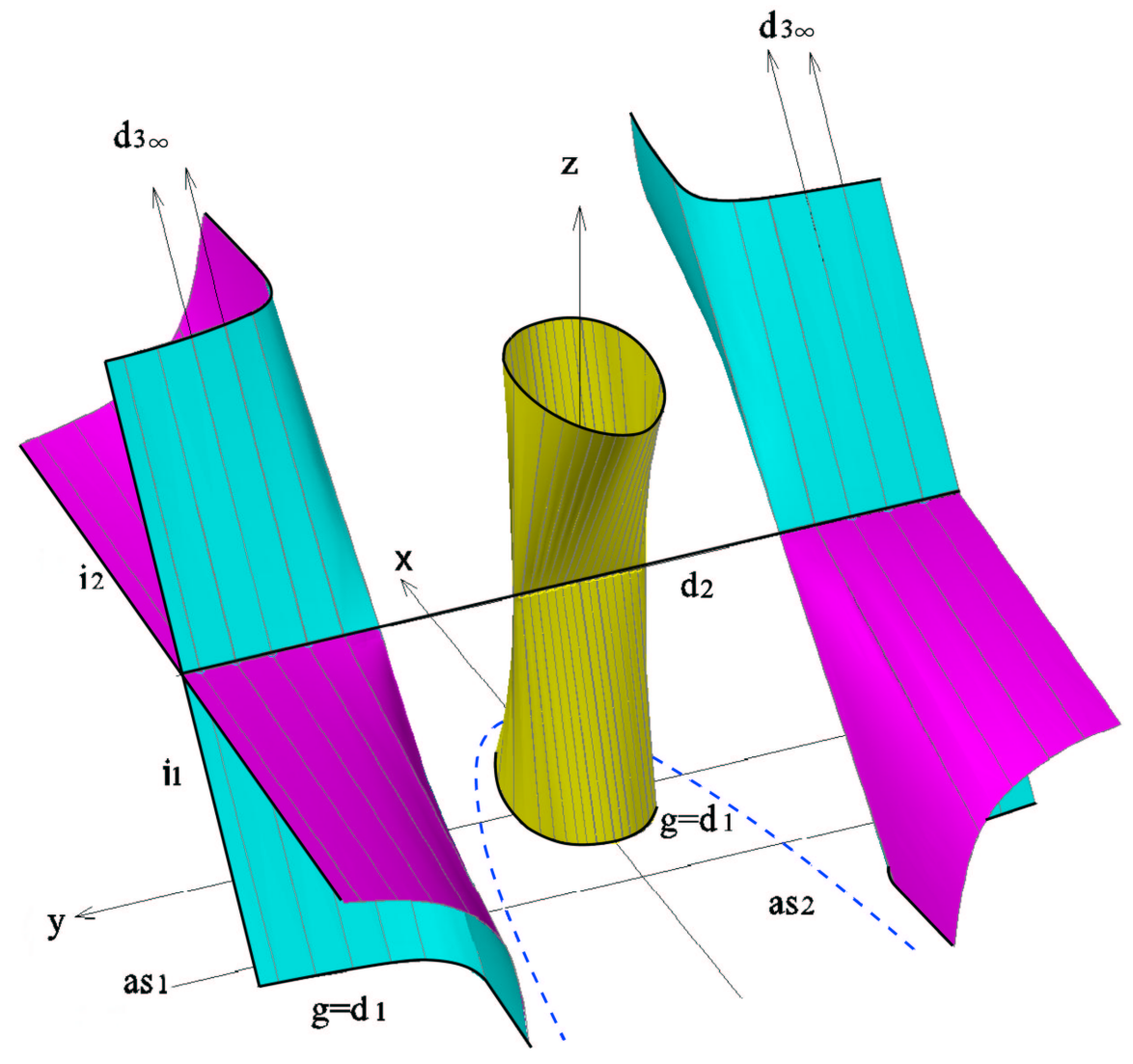

Figure 1 .

Each generatrix of the surface will intersect all three directrices [7]. The surface obtained in this manner will be quintic surface [11], degenerated into a conoid of the fourth order, as shown at Figure 1, with the equation [11]:

$$
\left(a^{2} y^{2}+d^{2} y^{2}-a^{2} b^{2}\right)(z-h)^{2}-2 d h x y^{2}(z-h)+b^{2} h^{2} x^{2}=0
$$

and a plane:

$$
z=h \text {. }
$$

Thus, we give an explanation of origination and the order of the surface, from the aspect of Projective Geometry.

1. As every plane $F_{1}$ parallel to the plane $x-z$, which will imply generatrices of the surface, intersects the plane $x-y$ of the curve $g$ by a straight line parallel to the $x$ axes [4], and each straight line must intersect the cubic curve by three points 
$X_{1}, X_{2}$ and $X_{3}$, we conclude that in each point of the directrix $d_{2}$ there intersect three generatrices: $i_{1}, i_{2}$, and $i_{3}$, of the surface. Therefore, the directrix $d_{2}$ will be triple line of the surface [5].

2. Because of the symmetry of the curve $g$ in relation to the $x$ axes, pair of generatrices of the conoid will intersect in the same point of the infinite directrix $d_{3}$, so it will be double line of the surface.

3. Cubic curve $g=d_{1}$ has two asymptotes $a s_{1}$ and $a s_{2}$, whereat the asymptote $a s_{1}$ is parallel to the $y$ axes, and $a s_{2}$ is parabola [9] with $x$ axes. Therefore, the cubic curve $g$ has two infinite points, $X_{\infty}$ and $Y_{\infty}$, of the axes $x$ and $y$.

4. $X_{\infty}$ represents the infinite vertex of the parabola $a s_{2}$. Every generatrix $i_{3}$ of the surface passes through this point forming the plane $H_{1}$, jointly with the directrix $d_{2} . Y_{\infty}$ is a point of tangency of curve $g=d_{1}$ and asymptote $a s_{1}$. At the same time, it is a triple point of intersection of the directrix $d_{2}$ and the plane $x-y$.

5. Points $X_{\infty}$ and $Y_{\infty}$ belong to infinite straight line $q_{\infty}$ of the plane $x-y$, which passes two times through those points. Straight line $q_{\infty}$ represents two overlapped generatrices $i_{2}$ and $i_{3}$ which pass through the infinite point $Y_{\infty}$ and are tangents to the vertex $X_{\infty}$ of the parabolic asymptote $a s_{2}$ (and also the curve $g$ ) at two immeasurable close points, $X_{2}$ and $X_{3}$, which are collinear to the point $Y_{\infty}$. Therefore, generatrices $i_{2}\left(X_{2 \infty} Y_{\infty}\right)$ and $i_{3}\left(X_{3 \infty} Y_{\infty}\right)$ will be identical, and so the straight line $q_{\infty}$ will be also the double line. Generatrix $i_{1}$ will be separated, so in the infinite plane $\omega \infty$, there will lie five lines: double line $d_{3 \infty}$, double line $q_{\infty}$, and the line $i_{1 \infty}$.

6. Plane $x-y$ intersects the conoidal surface by the initial cubic curve $g$ and by the infinite double straight line $q_{\infty}$, which indicates that it is the case of the surface of fifth degree [11].

7. Considering that the generatrix $i_{3}$ is a straight line that belongs to the pencil of straight lines through the point $X_{\infty}$, which constitutes, along with directrix $d_{2}$, the plane $H_{1}$, the surface will be degenerated to the conoid and the plane $H_{1}$. By eliminating the plane, the doubleness of the line $q_{\infty}$ subdues, as well as the tripleness of the directrix $d_{2}$, so the conoidal surface remains of the fourth order.

8. The horizontal plane $H_{1}$, at the elevation $z=h$ of the directrix $d_{2}$ is asymptotic plane of the surface. It is tangential plane onto the infinite double straight line $q_{\infty}$. Besides, there will exist yet another asymptotic plane, a skew plane $R$, which is determined by the directrix $d_{2}$ and the asymptote $a s_{1}$ of the curve $g=d_{1}$, as two parallel lines, and is tangential the conoid by the infinite straight line $r_{\infty}=i_{1}$.

9. The infinite plane of the space $\omega_{\infty}$, intersects the conoid by one double straight line $d_{3}$ and also by the remaining straight lines $q_{\infty}$, and $r_{\infty}$, which proves that it is the surface of fourth order.

Mathematical explanation of the originating surface, initially of the fifth order is easily obtained by simple multiplication of the equation of the fourth order (24) by factor $z-h$. The equation derived in this manner: $\left(a^{2} y^{2}+d^{2} y^{2}-a^{2} b^{2}\right)(z-$ $h)^{3}-2 d h x y^{2}(z-h)^{2}+b^{2} h^{2} x^{2}(z-h)=0$, decomposes to an equation of the fourth 
order (24) and an equation of a plane (25), as it is given in the commentary of the Projective Geometry.

Let us consider if it is possible for the plane section of the surface (24) to be a non-degenerated conic [11]. Let us emphasize that this problem is equivalent to the following system:

$$
\left\{\begin{array}{c}
(26 / i)\left(a^{2} y^{2}+d^{2} y^{2}-a^{2} b^{2}\right)(z-h)^{2}-2 d h x y^{2}(z-h)+b^{2} h^{2} x^{2}=0 \\
(26 / i i) A x+B y+C z+D=0
\end{array}\right\}
$$

which has solution by non-degenerated conic, for some real constants $A, B, C, D$. Using technique of Groebner bases we will prove that is not possible. The following statements are true:

Lemma 3.1. (i) Plane $x=\alpha$, for $\alpha \in \mathbf{R} \backslash\{0\}$, has the intersection with the surface $(26 / i)$ by the curve of the fourth order. Plane $x=0$ has the intersection with the surface $(26 / i)$ by $x=0, z=h(|y| \leq b \vee|y| \geq a b / d)$ or $x=0, y= \pm a b / \sqrt{a^{2}+d^{2}}$.

(ii) Plane $y=\beta$, for $\beta \in \mathbf{R}$, has intersection with the surface $(26 / i)$ iff:

$$
|\beta| \leq b \quad \vee \quad|\beta| \geq \frac{a b}{d} \quad\left(\frac{a b}{d}>b\right) ;
$$

then the intersection is presented as two straight lines (which determine degenerated conic).

(iii) Plane $z=\gamma$, for $\gamma \in \mathbf{R} \backslash\{h\}$, has the intersection with the surface $(26 / i)$ by the curve of the third order. Plane $z=h$ has the intersection with the surface $(26 / i)$ by $x=0, z=h(|y| \leq b \vee|y| \geq a b / d)$.

Proof. (i) By substitution $x=\alpha \in \mathbf{R}$ in $(26 / i)$ we obtained:

$$
\begin{aligned}
& \left(y^{2}\left(a^{2}+d^{2}\right)-a^{2} b^{2}\right)(z-h)^{2}-2 h d \alpha y^{2}(z-h)+h^{2} b^{2} \alpha^{2}=0 \\
\Longleftrightarrow & (z-h)\left(a^{2} b^{2}-\left(a^{2}+d^{2}\right) y^{2}\right)=\left(-d y^{2} \pm \sqrt{\left(b^{2}-y^{2}\right)\left(a^{2} b^{2}-d^{2} y^{2}\right)}\right) h \alpha .
\end{aligned}
$$

Based on $a, d>0$ we can conclude that the intersection is one algebraic curve of the forth order if $\alpha \neq 0$. If $\alpha=0(\Longleftrightarrow x=0)$, then from the previous equation follows $x=0, z=h(|y| \leq b \vee|y| \geq a b / d)$ or $x=0, y= \pm a b / \sqrt{a^{2}+d^{2}}$.

(ii) By substitution $y=\beta \in \mathbf{R}$ in $(26 / i)$ we obtained:

$$
\begin{aligned}
& b^{2} h^{2} x^{2}+\left(a^{2} \beta^{2}+d^{2} \beta^{2}-a^{2} b^{2}\right) z^{2}-2 d h \beta^{2} x z+ \\
& 2 d h^{2} \beta^{2} x+\left(2 a^{2} b^{2} h-2 d^{2} h \beta^{2}-2 a^{2} h \beta^{2}\right) z+ \\
& \left(a^{2} h^{2} \beta^{2}+d^{2} h^{2} \beta^{2}-a^{2} b^{2} h^{2}\right)=0 .
\end{aligned}
$$

If we solve the previous second order equation by $x$, from discriminant, we obtain the condition (27) for existence of the intersection via two straight lines:

$$
x= \pm \frac{\beta^{2} d+\sqrt{\left(b^{2}-\beta^{2}\right)\left(a^{2} b^{2}-d^{2} \beta^{2}\right)}}{b^{2} h}(-z+h) .
$$


(iii) By substitution $z=\gamma \in \mathbf{R}$ in $(26 / i)$ we obtained:

$$
\begin{aligned}
& b^{2} h^{2} x^{2}+2 d h(h-\gamma)^{2} x y^{2}+\left(a^{2}+d^{2}\right)(h-\gamma) y^{2}-a^{2} b^{2}(h-\gamma)^{2}=0 \\
\Longleftrightarrow & x= \pm \frac{\sqrt{\left(b^{2}-y^{2}\right)\left(a^{2} b^{2}-d^{2} y^{2}\right)}-y^{2} d}{b^{2} h}(h-\gamma) .
\end{aligned}
$$

Based on $d, h>0$ we can conclude that the intersection is one algebraic curve of the third order iff $\gamma \neq h$. If $\gamma=h(\Longleftrightarrow z=h)$, then from the previous equation follows $z=h, x=0(|y| \leq b \vee|y| \geq a b / d)$.

Remark 3.2. Starting from geometrical definition of this type of conoid, the cases (i) and (iii) will always have real plane sections.

Theorem 3.3. If the solution of the system (26) exists then it is not a nondegenerated conic.

Proof. First we assume that $C \neq 0$. Then by substitution $z=-\frac{A}{C} x-\frac{B}{C} y-\frac{D}{C}$ in $(26 / i)$ we obtain $x-y$ projection of a possible intersection:

$$
\begin{aligned}
& \left(\frac{A^{2} a^{2}}{C^{2}}+\frac{A^{2} d^{2}}{C^{2}}+\frac{2 d h A}{C}\right) x^{2} y^{2}+\left(\frac{2 A B d^{2}}{C^{2}}+\frac{2 d h B}{C}+\frac{2 A B a^{2}}{C^{2}}\right) x y^{3}+ \\
& \left(\frac{B^{2} d^{2}}{C^{2}}+\frac{B^{2} a^{2}}{C^{2}}\right) y^{4}+\left(\frac{2 A h a^{2}}{C}+\frac{2 A D d^{2}}{C^{2}}+\frac{2 A h d^{2}}{C}+\frac{2 d h D}{C}+2 d h^{2}+\right. \\
& \left.\frac{2 A D a^{2}}{C^{2}}\right) x y^{2}+\left(\frac{2 B D d^{2}}{C^{2}}+\frac{2 B D a^{2}}{C^{2}}+\frac{2 B h a^{2}}{C}+\frac{2 B h d^{2}}{C}\right) y^{3}+ \\
& \left(h^{2} b^{2}-\frac{A^{2} a^{2} b^{2}}{C^{2}}\right) x^{2}+\frac{2 A B a^{2} b^{2}}{C^{2}} x y+\left(\frac{2 D h a^{2}}{C}+h^{2} a^{2}-\frac{B^{2} a^{2} b^{2}}{C^{2}}+\right. \\
& \left.\frac{D^{2} a^{2}}{C^{2}}+h^{2} d^{2}+\frac{2 D h d^{2}}{C}+\frac{D^{2} d^{2}}{C^{2}}\right) y^{2}-\left(\frac{2 A D a^{2} b^{2}}{C^{2}}+\frac{2 A h a^{2} b^{2}}{C}\right) x- \\
& \left(\frac{2 B h a^{2} b^{2}}{C}+\frac{2 B D a^{2} b^{2}}{C^{2}}\right) y-h^{2} a^{2} b^{2}-\frac{2 D h a^{2} b^{2}}{C}-\frac{D^{2} a^{2} b^{2}}{C^{2}}=0 .
\end{aligned}
$$

The previous equation is algebraic equation of the second order iff:

$$
\begin{aligned}
& \frac{A^{2} a^{2}}{C^{2}}+\frac{A^{2} d^{2}}{C^{2}}+\frac{2 d h A}{C}=0 \wedge \\
& \frac{2 A B d^{2}}{C^{2}}+\frac{2 d h B}{C}+\frac{2 A B a^{2}}{C^{2}}=0 \wedge \\
& \frac{B^{2} d^{2}}{C^{2}}+\frac{B^{2} a^{2}}{C^{2}}=0 \wedge \\
& \frac{2 A h a^{2}}{C}+\frac{2 A D d^{2}}{C^{2}}+\frac{2 A h d^{2}}{C}+\frac{2 d h D}{C}+2 d h^{2}+\frac{2 A D a^{2}}{C^{2}}=0 \wedge \\
& \frac{2 B D d^{2}}{C^{2}}+\frac{2 B D a^{2}}{C^{2}}+\frac{2 B h a^{2}}{C}+\frac{2 B h d^{2}}{C}=0 .
\end{aligned}
$$

Solving previous system, via Maple, we obtain two subcases:

$\mathbf{1}^{\mathbf{0}} . A=0, B=0, C=p, D=-p h \quad \vee \quad \mathbf{2}^{\mathbf{0}} \cdot A=q, B=0, C=-\frac{a^{2}+d^{2}}{2 d h} q, D=\frac{a^{2}+d^{2}}{2 d} q$ for $p, q \in \mathbf{R} \backslash\{0\}$. Therefore: 
$\mathbf{1}^{\mathbf{0}}$. The system (26):

$$
\left\{\begin{array}{c}
\left(a^{2} y^{2}+d^{2} y^{2}-a^{2} b^{2}\right)(z-h)^{2}-2 d h x y^{2}(z-h)+b^{2} h^{2} x^{2}=0, \\
z-h=0 ;
\end{array}\right\}
$$

has the reduced Groebner basis (related to lexicographic order) in the following form:

$$
g_{11}:=x^{2} \quad \wedge \quad g_{12}:=z-h .
$$

Hence, the system (26) has the intersection by directrix $d_{2}$ and this intersection is not non-degenerated conic.

$\mathbf{2}^{\mathbf{0}}$. The system (26):

$$
\left\{\begin{array}{c}
\left(a^{2} y^{2}+d^{2} y^{2}-a^{2} b^{2}\right)(z-h)^{2}-2 d h x y^{2}(z-h)+b^{2} h^{2} x^{2}=0, \\
2 d h x-\left(a^{2}+d^{2}\right) z+h\left(a^{2}+d^{2}\right)=0 ;
\end{array}\right\}
$$

has the reduced Groebner basis (related to lexicographic order) in the following form:

$$
g_{21}:=z^{2}-2 z h+h^{2} \wedge g_{22}:=2 d h x-\left(a^{2}+d^{2}\right) z+h\left(a^{2}+d^{2}\right) .
$$

Hence, the system (26) has intersection by directrix $d_{2}$ and this intersection is not non-degenerated conic.

Let $C=0$. We can consider two cases: $B=0$ or $B \neq 0$. If $B=0$ subcase $A=0$ does not lead to a planar intersection. If $B=0$ subcase $A \neq 0$ is considered in the Lemma 3.1. (case $(i)$ ). Finally, let us observe case $B \neq 0$. By substitution of $y=-\frac{A}{B} x-\frac{D}{B}$ in $(26 / i)$ we obtain $x-z$ projection of a possible intersection:

$$
\begin{aligned}
& -\frac{2 d h A^{2}}{B^{2}} x^{3} z+\left(\frac{a^{2} A^{2}}{B^{2}}+\frac{d^{2} A^{2}}{B^{2}}\right) x^{2} z^{2}+\frac{2 d h^{2} A^{2}}{B^{2}} x^{3}-\left(\frac{4 d h A D}{B^{2}}+\right. \\
& \left.\frac{2 h a^{2} A^{2}}{B^{2}}+\frac{2 h d^{2} A^{2}}{B^{2}}\right) x^{2} z+\left(\frac{2 a^{2} A D}{B^{2}}+\frac{2 d^{2} A D}{B^{2}}\right) x z^{2}+\left(\frac{4 d h^{2} A D}{B^{2}}+\right. \\
& \left.\frac{h^{2} a^{2} A^{2}}{B^{2}}+h^{2} b^{2}+\frac{h^{2} d^{2} A^{2}}{B^{2}}\right) x^{2}-\left(\frac{2 d h D}{B^{2}}+\frac{4 h d^{2} A D}{B^{2}}+\frac{4 h a^{2} A D}{B^{2}}\right) x z+ \\
& \left(\frac{a^{2} D}{B^{2}}-a^{2} b^{2}+\frac{d^{2} D}{B^{2}}\right) z^{2}+\left(\frac{2 h^{2} d^{2} A D}{B^{2}}+\frac{2 d h^{2} D}{B^{2}}+\frac{2 h^{2} a^{2} A D}{B^{2}}\right) x+ \\
& \left(2 h a^{2} b^{2}-\frac{2 h a^{2} D}{B^{2}}-\frac{2 h d^{2} D}{B^{2}}\right) z+\left(\frac{h^{2} d^{2} D}{B^{2}}+\frac{h^{2} a^{2} D}{B^{2}}-h^{2} a^{2} b^{2}\right)=0 .
\end{aligned}
$$

Therefore $A=0$ and we obtain the case (ii) from the Lemma 3.1.

Remark 3.4. Let us emphasize that we may consider possible factorizations of polynomials (28) and (29) in purpose of determining a type of section by non-degenerated conic as a part of plane intersection of this egg curve based conoid. 


\section{CONCLUSIONS}

In the paper an application of Groebner bases is considered, in order to define planarity of two surfaces intersection. An egg curve based conoid is analyzed in particular, from the aspect of Projective Geometry. Its plane sections are analyzed from the aspect of Analytic Geometry. Using the example of the egg curve based conoid, a method is developed, based on the technique of Groebner bases, which enables investigation of existence of the assigned type of planar section. Based on the presented method, in some cases, it is possible to determine even for the other algebraic surfaces if they would imply a planar section that consists of nondegenerated conics.

\section{REFERENCES}

[1] R. FröBerg: An Introduction to Gröbner Bases, John Wiley \& Sons, 1997.

[2] D. A. Cox, J. B. Little, D. O'Shea: Ideals, Varieties, and Algorithms: An Introduction to Computational Algebraic Geometry and Commutative Algebra, Third Edition, Springer, 2007.

[3] L. Dovniković: Nacrtno geometrijska obrada i klasifikacija ravnih krivih 3. reda (Descriptive Geometrical Teatment And Classification Of Plane Curves Of The Third Order), Matica srpska, Zbornik za prirodne nauke, sv. 52, 1977, str. 181-185.

[4] A. GraY: Modern Differential Geometry of Curves and Surfaces with Mathematica, Second Edition, CRC Press, 1998.

[5] S. GorJanc: Quartics in E3 which Have a Triple Point and Touch the Plane at Infinity through the Absolute Conic, Mathematical Communication 9 (2004), $67-78$.

[6] V. Y. RovenskiI: Geometry of Curves and Surfaces With Maple, Springer, 2000.

[7] V. Sbutega, S. ŽIvanović: Projektivna geometrija osnovnih likova prve vrste $i$ njihovih tvorevina (Projective Geometry of Elementary Figures of the first Sort And Their Products), Arhitektonski fakultet, Beograd, 1986.

[8] Lu. Velimirović, M. Stanković. G. Radivojević: Modeling Conoid Surfaces, Facta Universitatis, Series: Architecture and Civil Engineering, Vol. 2, No 4, 2002, 261 - 266.

[9] S. J. Wilson: A Gallery of Cubic Plane Curves, from Plane Curve Panoramas (http://staff.jccc.net/swilson/planecurves/cubics.htm)

[10] L. Dovniković: Zapisi iz teorije krivih $i$ povri, skripta za poslediplomske studije, Arhitektonski fakultet, Beograd 1986. 
[11] M. Obradović, M. Petrović: The Spatial Interpretation of the Hügelschäffer's Construction of the Egg Curve, MoNGeometrija 2008, Proceedings, pp. 222-231, 24-th national and 1-st international convenction on Descriptive Geomtry and Engineering Graphics, Vrnjačka Banja 2008.

[12] B. MalešEvić: Applications of Groebner bases in computer graphics, MoNGeometrija 2008, Proceedings, pp. 180-186, 24-th national and 1-st international convenction on Descriptive Geomtry and Engineering Graphics, Vrnjačka Banja 2008.

[13] A. Heck: Bird's-eye view of Gröbner Bases, Nuclear Inst. and Methods in Physics Research A 389 (1997) 16 - 21.

(http://staff.science.uva.nl/ heck/AIHENP96/groebnerbasis.pdf )

[14] A. Heck: Introduction to Maple, Third Edition, Springer 2003.

[15] E. Roanes-Lozano, E. Roanes-Macías, L.M. Laita: Some applications of Gröbner bases, Computing in Science and Engineering, 6 (3): 56-60, May/Jun 2004.

[16] R. KARP: Great Algorithms, CS Cousre 294-5, spring 2006, Berkeley. ( http://www.cs.berkeley.edu/ ${ }^{k}$ karp/greatalgo/)

${ }^{*}$ Faculty of Electrical Engineering, Faculty of Civil Engineering; Belgrade; Serbia E-mails: malesevic@etf.bg.ac.yu, marijao@grf.bg.ac.yu 